\title{
3 Research Soure \\ Examining cell-to-cell contacts to predict the efficacy of CAR immunotherapy
}

Dongfang Liu

Saiaditya Badeti

Gianpietro Dotti

Jie-gen Jiang

He Wang

James Dermody

Patricia Soteropoulos

Deanna Streck

Raymond B. Birge

Chen Liu

\section{Video Byte}

Keywords: immunotherapy, CAR-T cell, CAR, chimeric antigen receptor, immune response, cancer, blood, Cell Communication and Signaling

Posted Date: November 11th, 2020

DOl: https://doi.org/10.21203/rs.3.rs-106058/v1

License: (c) (1) This work is licensed under a Creative Commons Attribution 4.0 International License. Read Full License 


\section{Abstract}

Chimeric Antigen Receptor (CAR) immunotherapy is a promising new technique for precisely targeting tumor cells. The technique works by using the patient's own cells to express a molecule that combines tumor antigen specificity with immune cell activation. Doctors have already had some success treating many types of refractory blood cancers. Unfortunately, conventional tools are limited when it comes to evaluating the efficacy and safety of CAR treatment, and the potential for high toxicity makes it critical to identify patients who will benefit from the treatment. To address this, researchers have developed a new method of predicting the efficacy and toxicity of CAR immunotherapy. The Synapse Predicts Efficacy (SPE) system begins by measuring the contact site between immune cells and tumor cells - the immunological synapse. The system relies on microscopic measurements of immunological synapse quality - particularly effective polarization of the immune cell to the antigen. These measurements can be used to predict whether CAR-modified immune cells will be effective against cancer cells. Although studies with larger populations are underway, SPE may help determine which patients will benefit most from CAR immunotherapy, optimizing patient selection for clinical trials and helping to improve the prognosis for patients with blood cancers. 